\title{
Improvement of SIMPLE Algorithm in Centrifugal Pump for CFD
}

\author{
Liu Houlin, Ren Yun *, Wu Denghao, Yang Hongbin and Tan Minggao \\ Research Center of Fluid Machinery Engineering and Technology, Jiangsu University, China
}

Received: 17 Jan. 2013, Revised: 20 May. 2013, Accepted: 21 May. 2013

Published online: 1 Sep. 2013

\begin{abstract}
In this paper, SIMPLED algorithm, an improvement algorithm of SIMPLE, was developed, which is suitable for simulation of inner flow in centrifugal pumps. Based on the open source code OpenFOAM, a new solver was compiled by using new algorithm based on the original solver. To compare the efficiency and accuracy of SIMPLE and SIMPLED, the two algorithms were used to simulate the inner flow of centrifugal pumps with different specific speeds under the design condition. The results showed that under the same convergence criterion, the iteration time of SIMPLED was almost the same as SIMPLE. However, the head prediction discrepancy of the former was about $3 \%$ smaller than that of the latter, and the efficiency prediction discrepancy of the pumps, the decrease was about $2 \%$. In order to further study the applicability of SIMPLED algorithm, the flow in a medium specific speed centrifugal pump under three flow rate conditions was simulated by these two algorithms. By comparison, it was found out that the SIMPLED algorithm was also more accurate than the SIMPLE algorithm for inner flow simulation at off-design conditions. Therefore, SIMPLED algorithm is a new efficient and exact modification of SIMPLE algorithm.
\end{abstract}

Keywords: Numerical simulation, SIMPLE algorithm, OpenFOAM, Centrifugal pump

\section{Introduction}

Understanding of the inner flow rules of centrifugal pump is one of the most complicated flow problems in fluid mechanics. Meanwhile, as an indispensable part of numerical simulation, the computational method is one of the important components in computational fluid dynamics (CFD)[1,2,3]. Currently, the pressure correction method is one of the most popular computational methods used in engineering. The semi-implicit method for pressure-linked equations, namely SIMPLE algorithm, is widely used to calculate the velocity and pressure, because there is no independent governing equation for pressure in the pressure correction method[4]. Besides, it also greatly promotes the advance of SIMPLE algorithm, and the most famous improved algorithms include SIMPLER algorithm[5] and SIMPLEC algorithm[6]. All of those algorithms have successfully solved the velocity-pressure decoupling problem with staggered grids. However, the definite drawbacks of staggered grids are that it requires complicated interpolations, complex computer programs and large EMS (Expanded Memory System)[7,8,9]. Therefore, collocated grid technique was firstly proposed by Rhie and Chow[10] in 1983. Then, it was confirmed again by Peric[11] in 1988. To ensure the coupling between pressure and velocity, the cell-face velocities were evaluated by MI (Momentum Interpolation) method[12], which is popular within the recent research. The velocities are stored on both cell faces and nodes, because the cell-face velocities are interpolated by momentum interpolation practice rather than being linearly interpolated. Date[13] showed that the above interpolation method was unnecessary, and it might predict spurious cell-face velocities when local variation of pressure departed considerably from linearity. A new pressure correction equation on structured collocated meshes in Cartesian coordinate system was proposed by Date. In this case, smooth pressure distribution can be predicted even on a coarse grid. Furthermore, it simplified programming procedure and used less EMS memory. Therefore, this practice was a more economic algorithm. In such a circumstance, the cell-face velocities and pressure gradient were both represented by the arithmetic mean interpolation. It has been gradually regarded by researchers in recent studies[14,15]. However, the algorithm proposed by Date will lower the numerical

\footnotetext{
${ }^{*}$ Corresponding author e-mail: renyun_ry@ hotmail.com
} 
simulation accuracy of centrifugal pump while based on unstructured meshes, because that the cell face velocities and pressure term were adopted by arithmetic average value.

In this paper, the pressure correction algorithm proposed by Date is applied on unstructured collocated grids. Simultaneously, focusing on the inner flow simulation of centrifugal pump, new methods dealing with the cell-face velocities and pressure gradient evaluation are reconsidered. Then, a new solver with the improvement of SIMPLE algorithm, namely SIMPLED algorithm, is gained. Finally, the inner flow of centrifugal pump is simulated both by the new algorithm and the original one.

\section{Improvement on SIMPLE Algorithm}

\subsection{Introduction of SIMPLED algorithm}

The difference between SIMPLED and SIMPLE algorithm is in the pressure equation to be solved. In this algorithm, the initial pressure values and its update are completed by solving the Poisson equations separately. It is mainly used to solve incompressible flow field, which was proposed by Harlow and Welch in 1965[16]. The basic idea of the method is to take the Poisson equation instead of the continuity equation. Then, solve it together with momentum equation. The key point is to ensure that the condition of mass conservation is required. To introduce the main content of the method briefly, a two dimensional flow field was used as an example. The Poisson equation is obtained by solving the partial derivatives and then the additional operation on the governing equations of the two velocity components in incompressible flow field.

Convert the discrete momentum equations into vector form:

$$
a_{P} U_{P}=H\left(U_{P}\right)-\nabla p_{P},
$$

Where $H\left(U_{p}\right)=-\sum_{N} a_{N} U_{N} ; a_{P}$ is the primarily node coefficient; $a_{N}$ the adjacent node coefficient which meet the relation: $a_{P}=-\sum_{N} a_{N}$; the source item is included in $H\left(U_{P}\right)$.

Discrete form of continuity equation is as follow:

$$
\nabla \cdot U=\sum_{f} S_{f} \cdot U_{f}=0
$$

From equation (1), we can obtain the following function:

$$
U_{P}=\frac{H\left(U_{P}\right)}{a_{P}}-\frac{\nabla p_{P}}{a_{P}}
$$

Like equation (3), the cell-face velocities are calculated via the momentum-interpolation practice given as follows:

$$
U_{f}=\left(\frac{H\left(U_{P}\right)}{a_{P}}\right)_{f}-\left(\frac{1}{a_{P}}\right)_{f}\left(\nabla p_{P}\right)_{f}
$$

Where $U_{f}$ is the cell face velocity.

In Date's algorithm, the cell face velocity was adopted by arithmetic average value. However, while finite volume method is applied to discretize the Navier-Stokes equations, the interpolation method of velocities on the cell faces will have influence on the computational accuracy. Therefore, the interpolation method with area as weighted factor is adopted. That is, interpolating the value at the cell center onto the face, then, the cell face velocity is interpolated by average weighted interpolation with the area as weighted factor. It can limit the velocity field and pressure field to a rational range. The equation is as follows:

$$
\phi_{f}^{\prime}=\frac{\sum_{f} S_{f} \phi_{f}}{\sum_{f} S_{f}}
$$

Taking into account the calculation accuracy of the velocity, an empirical coefficient of the average velocity above is adopted and a new cell-face velocity obtained above, as follows:

$$
\bar{U}_{f}=K_{1} \bar{U}_{f}^{\prime}
$$

Where, $K_{1}$ is empirical coefficient. The average cell-face velocity $\bar{U}_{f}$ can then be obtained by interpolating the cellcenter average velocity onto the cell-face. The mass flux through the cell-face is:

$$
F=S_{f} \cdot \bar{U}_{f}
$$

Substituting equation (3) into (2), the pressure equation is obtained

$$
\nabla \cdot\left(\frac{1}{a_{P}} \nabla p_{P}\right)=\nabla \cdot\left(\frac{H\left(U_{P}\right)}{a_{P}}\right)=\sum_{f} S_{f} \cdot \bar{U}_{f}
$$

Another key point to solving the incompressible flow $\mathrm{N}-\mathrm{S}$ equations is to figure out the coupling of pressure and velocity and the update of the pressure field. The method presented by Date overcomes the defect of momentum interpolation method when pressure in general departs from linearity. Both [13] and [17] introduced the theory of Date's method, demonstrated the advantages of this method, and derived the pressure correction equation made by Date's method:

$$
\frac{\partial}{\partial x}\left[D_{x} \frac{\partial p_{p}{ }^{\prime}}{\partial x}\right]+\frac{\partial}{\partial y}\left[D_{y} \frac{\partial p_{p}{ }^{\prime}}{\partial y}\right]=\frac{\partial\left(\rho \bar{u}^{*}\right)}{\partial x}+\frac{\partial\left(\rho \bar{v}^{*}\right)}{\partial y}
$$

The boundary conditions are as follows:

$$
\begin{gathered}
\left.\frac{\partial p_{P}{ }^{\prime}}{\partial n}\right|_{\partial \Omega}=0 \\
D_{x}=\frac{\rho \alpha \Delta}{a_{P}^{u}} \quad D_{y}=\frac{\rho \alpha \Delta}{a_{P}^{v}}
\end{gathered}
$$

Where, $a_{P}^{u}, a_{P}^{v}$ are principal diagonal coefficients of $u$ and $v$ algebraic equations respectively. The form of equation (9) is the same as the differential form of the momentum interpolation method. However, Date introduced the cell 
average pressure. In addition, it is usual to take the inversion of the distance between master node to its neighborhood as weighted value while calculating the averaging scalar at the cell center average method on unstructured meshes[18]. In order to reduce the computational time, inverse weighted average method is usually adopted while averaging nodal pressure variables. A correction pressure calculation is as follows:

$$
p_{m, P}{ }^{\prime}=p_{P}^{\prime}-\frac{1}{2}\left(p_{P}^{*}-\bar{p}_{P}{ }^{*}\right)
$$

Where, $p_{m, P} /$ is the actual pressure correction; $p_{P}$ is the value of the previous iteration; $\bar{p}_{P}{ }^{*}$ is the average pressure value of the previous iteration, following the equation below:

$$
\bar{p}_{P}^{*}=\frac{1}{2}\left(\frac{\Delta x^{+} p_{W}^{*}+\Delta x^{-} p_{E}^{*}}{\Delta x^{+}+\Delta x^{-}}+\frac{\Delta y^{+} p_{S}^{*}+\Delta y^{-} p_{N}^{*}}{\Delta y^{+}+\Delta y^{-}}\right)
$$

Both equation (12) and (13) solve the problem that the pressure variation is spatially nonlinear, which are not suitable to use in the original momentum interpolation method. Furthermore, the Date's method was realized based on structured meshes. However, for the unstructured meshes, dealing with $\bar{p}_{P}{ }^{*}$ is more complex than that on structured meshes. In order to save the computing time, the following method introduced by [18] is employed:

$$
\bar{p}_{P}^{*}=\frac{\sum_{n} \frac{p_{n}^{*}}{d_{n}}}{\sum_{n} \frac{1}{d_{n}}}
$$

Where, $d_{n}$ is the distance between node $\mathrm{P}$ and its adjacent node; Simultaneously, equation (12) is multiplied by an empirical coefficient to accelerate the converging speed and to improve the convergent behavior, as follows:

$$
p_{m, P}{ }^{\prime}=K_{2}\left[p_{P}{ }^{\prime}-\frac{1}{2}\left(p_{P}{ }^{*}-\bar{p}_{P}{ }^{*}\right)\right]
$$

Here, $K_{2}$ is the empirical coefficient, the updates for pressure and velocity are as shown below:

$$
\begin{gathered}
p=p^{*}+\beta p_{m, P^{\prime}} \\
u=u^{*}-\left(\frac{\alpha \Delta}{a_{P}^{u}}\left|\frac{\partial p_{m, P^{\prime}}}{\partial x}\right|_{P}\right) \\
v=v^{*}-\left(\frac{\alpha \Delta}{a_{P}^{v}}\left|\frac{\partial p_{m, P^{\prime}}}{\partial y}\right|_{P}\right)
\end{gathered}
$$

Where, $\beta$ and $\alpha$ are respectively the relaxation factor for pressure and velocity calculation.

\subsection{Computational steps}

1.A pressure field $p^{*}$ is guessed.
2.Momentum equations are discretized and the nodes velocity components $u^{*}$ and $v^{*}$ are solved. Meanwhile, the coefficient $a_{P}$ and source term $b_{P}$ in equation (1) are also given.

3.The cell face velocities are calculated by the interpolation equation (4), then the cell face velocities are interpolated by average weighted interpolation.

4.The coefficients and source term in the pressure correction equation (9) are calculated, also with the correction pressure $p_{P}{ }^{\prime}$ on the nodes.

5.The average pressure value of the previous iteration is solved from equation (14), and the correction $p_{m, P}{ }^{\prime}$ is obtained at all nodes.

6.According to equation (16), (17) and (18), all the velocity components $u, v$ and pressure field $p$ are corrected.

7.Check whether the solutions converge or not. If they do not converged, return to step (2), using the corrected velocity field and pressure field as improved estimates for the next iteration, until all the resolutions converge.

\subsection{Coefficients selection}

\subsubsection{Model}

While calculating the inner flow of centrifugal pumps by the SIMPLED algorithm, it has been observed that the values of $K_{1}$ and $K_{2}$ have direct effect on the fluctuation of velocity and pressure. Thus, they also influence significantly the precision, stability and iteration time of simulation. Therefore, 6 pumps with different specific speeds were adopted. The structure parameters and experimental characteristics of those pumps are shown in Table 1. All of those models were generated by Pro/E. In order to reduce the impact by boundary conditions, a straight taper suction and an outlet pipe with about 4 times the diameter of the volute outlet are added. First of all, three pumps with different specific speeds were adopted to fix the value of the coefficients $K_{1}$ and $K_{2}$. Lastly, the coefficients $K 1$ and $K 2$ were verified by the remaining three pumps.

\subsubsection{Numerical methods}

SIMPLED algorithm is employed instead of SIMPLE algorithm in original solver, that is, MRFSimpleFoam solver. A new solver named MRFSimpledFoam in open source code OpenFOAM is set up. The geometries are meshed in hybrid grid by GAMBIT. In order to verify the results of numerical simulation, the grid independency was studied with 4 schemes before the simulation. Figure 1 shows the grids of the pumps used for the research. After the meshes are imported in OpenFOAM, the boundary conditions are as follows:

At the inlet, the constant uniform velocity with specific speed was adopted. 
Table 1: The structure parameters and experimental characteristics of centrifugal pumps

\begin{tabular}{|c|c|c|c|c|c|c|c|c|c|c|c|}
\hline \multirow[b]{2}{*}{ No. } & \multicolumn{5}{|c|}{ experimental characteristics } & \multicolumn{6}{|c|}{ structure parameters } \\
\hline & $\begin{array}{c}Q_{d} \\
/ \mathrm{m}^{3} \cdot \mathrm{h}^{-1}\end{array}$ & $\begin{array}{l}H \\
/ \mathrm{m}\end{array}$ & $\begin{array}{c}n \\
/ \mathrm{r} \cdot \min ^{-1}\end{array}$ & $n_{s}$ & $\begin{array}{l}\eta \\
1 \%\end{array}$ & $Z$ & $\begin{array}{c}D_{2} \\
/ \mathrm{mm}\end{array}$ & $\begin{array}{c}b_{2} \\
/ \mathrm{mm}\end{array}$ & $\begin{array}{l}\beta_{2} \\
/^{\circ}\end{array}$ & $\begin{array}{c}D_{3} \\
/ \mathrm{mm}\end{array}$ & $\begin{array}{c}b_{3} \\
/ \mathrm{mm}\end{array}$ \\
\hline 1 & 20.00 & 30.80 & 2900 & 60.40 & 64.00 & 6 & 162 & 7 & 32 & 176 & 20 \\
\hline 2 & 187.40 & 20.31 & 1450 & 126.20 & 90.06 & 6 & 268 & 25 & 29 & 280 & 48 \\
\hline 3 & 93.56 & 21.63 & 2900 & 170.10 & 84.75 & 6 & 140 & 26 & 34 & 150 & 42.5 \\
\hline 4 & 45.70 & 46.41 & 2900 & 67.10 & 75.82 & 6 & 200 & 10 & 31 & 210 & 22 \\
\hline 5 & 280.00 & 29.10 & 1450 & 117.80 & 82.50 & 6 & 315 & 30 & 27 & 342 & 60 \\
\hline 6 & 285.00 & 14.32 & 1485 & 280.15 & 88.00 & 6 & 246 & 39 & 25 & 246 & 60 \\
\hline
\end{tabular}

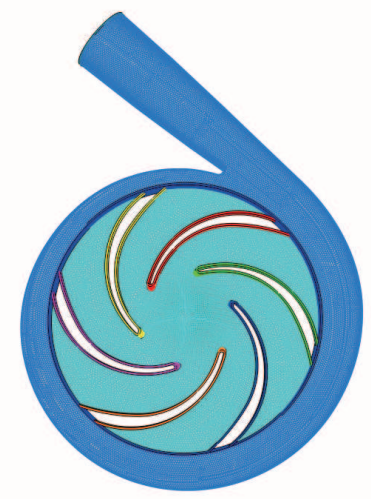

(a) pump1

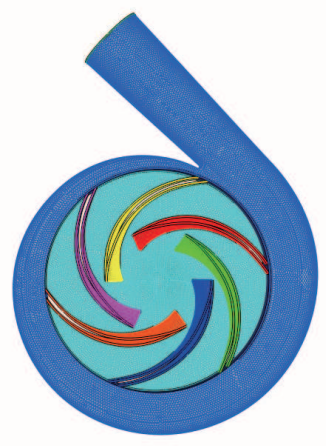

(d) pump4

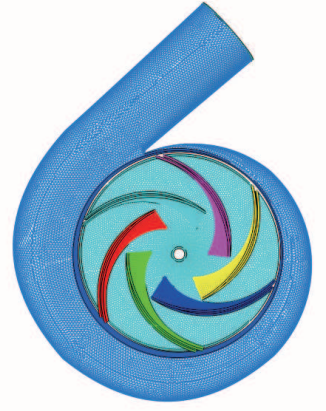

(b) pump2

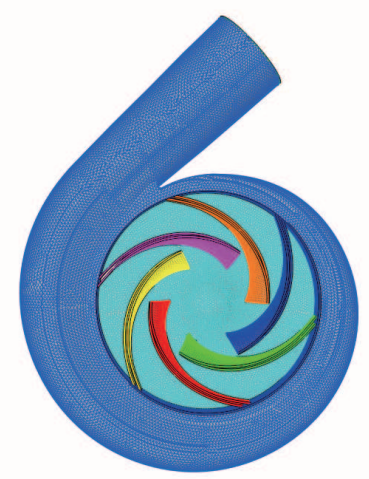

(e) pump5

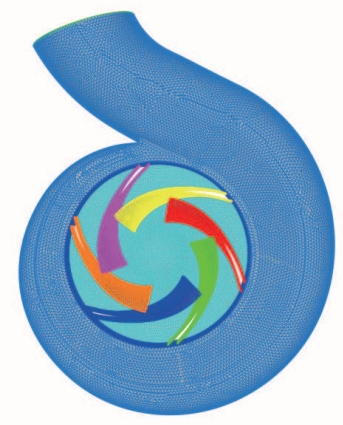

(c) pump3

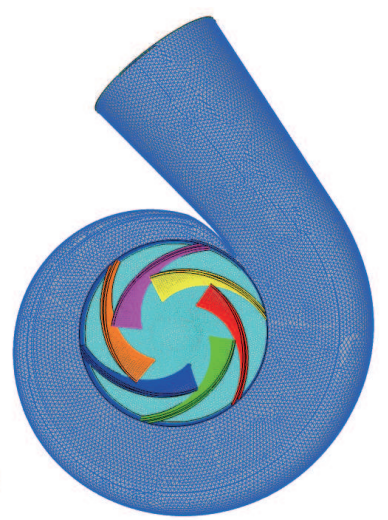

(f) pump6

Fig. 1: Grids

At the outlet, the constant pressure boundary was used with $p=0 P a$, and the rest value gradients are zero gradient.

Wall condition: On the solid wall, the no-slip wall boundary was used. Meantime, the wall function was used to define the flow near the wall.

In order to simulate the whole pump, the GGI method is needed to transmit the information between rotor and stator.
The specification of the computer used is CPU Pentium $2.5 \mathrm{GHz}$ with a 4GB RAM. The simulation is used OpenFOAM-1.5-dev code on SUSE Linux 11.1.

\subsubsection{Computational results}

The random search of the coefficients $K_{1}$ and $K_{2}$ among the pumps from No.1 to No.3 with different specific speeds are given, accompanied by the simulation data in Table 2. The optimization coefficients were made over a number of configurations. The best five configurations for 
Table 2: Simulation results of random research

\begin{tabular}{|c|c|c|c|c|c|c|c|c|c|c|}
\hline No. & $K_{1}$ & $K_{2}$ & $\begin{array}{l}H \\
/ \mathrm{m}\end{array}$ & $\begin{array}{c}M \\
/ \mathrm{N} \cdot \mathrm{m}\end{array}$ & $\begin{array}{l}\eta_{h} \\
/ \%\end{array}$ & $\begin{array}{l}\% \\
1 \%\end{array}$ & $\begin{array}{l}\Delta H \\
/ \%\end{array}$ & $\begin{array}{l}\Delta \eta \\
/ \%\end{array}$ & Iteration steps & Iteration time $/ \mathrm{h}$ \\
\hline \multirow{6}{*}{1} & \multicolumn{2}{|c|}{ SIMPLE } & 33.75 & 7.54 & 80.29 & 66.56 & 9.57 & 2.56 & 2113 & 10.16 \\
\hline & 0.900 & 0.6 & 39.03 & 8.47 & 82.64 & 69.35 & 26.71 & 5.35 & 3500 & 18.36 \\
\hline & 0.971 & 0.6 & 35.32 & 8.03 & 78.89 & 65.89 & 14.66 & 1.89 & 3500 & 17.92 \\
\hline & 1.042 & 0.9 & 32.41 & 7.55 & 77.01 & 63.91 & 5.24 & -0.09 & 1995 & 10.19 \\
\hline & 1.071 & 0.9 & 31.19 & 7.45 & 75.10 & 62.26 & 1.27 & -1.74 & 2085 & $10.06(\mathrm{~A})$ \\
\hline & 1.10 & 0.8 & 34.19 & 7.55 & 82.37 & 68.27 & 12.57 & 4.27 & 2113 & 10.25 \\
\hline & SIMF & & 19.42 & 74.43 & 87.72 & 81.30 & -4.36 & -5.21 & 675 & 3.09 \\
\hline \multirow{5}{*}{2} & 0.900 & 0.3 & 22.19 & 83.13 & 89.73 & 83.49 & 9.26 & -3.11 & 755 & 3.78 \\
\hline & 0.929 & 0.4 & 21.41 & 80.56 & 89.35 & 83.04 & 5.44 & -3.52 & 677 & 3.34 \\
\hline & 0.958 & 0.4 & 20.41 & 79.25 & 86.57 & 80.43 & 0.49 & -1.82 & 702 & 3.43(B) \\
\hline & 1.029 & 0.3 & 19.42 & 74.43 & 87.72 & 81.30 & -4.36 & -3.74 & 679 & 3.07 \\
\hline & 1.100 & 0.5 & 15.37 & 70.77 & 73.03 & 67.63 & -24.30 & -20.56 & 660 & 3.16 \\
\hline \multirow{6}{*}{3} & \multicolumn{2}{|c|}{ SIMPLE } & 22.40 & 20.11 & 93.45 & 88.46 & 3.56 & 3.17 & 1015 & 5.67 \\
\hline & 0.900 & 1.0 & 25.07 & 22.98 & 91.58 & 86.96 & 15.92 & 2.21 & 1499 & 8.86 \\
\hline & 0.971 & 1.0 & 22.91 & 22.25 & 86.41 & 82.04 & 5.92 & -2.71 & 1502 & 11.60 \\
\hline & 1.032 & 1.1 & 21.08 & 19.58 & 90.37 & 85.51 & -2.53 & 0.76 & 1180 & $6.34(\mathrm{C})$ \\
\hline & 1.071 & 1.1 & 22.33 & 21.79 & 85.97 & 81.59 & 3.21 & -3.16 & 1609 & 12.17 \\
\hline & 1.100 & 0.9 & 19.08 & 20.86 & 76.74 & 72.82 & -11.79 & -11.93 & 1398 & 10.85 \\
\hline
\end{tabular}

each pump are given in Table 2. For the selection of coefficients of each pump, the critical values of $K_{1}$ and $K_{2}$ were roughly calculated firstly. Then, the successive coefficients were given with dichotomy. In addition, the inner flow of centrifugal pumps is simulated separately with SIMPLE and SIMPLED algorithms with different coefficients. Moreover, the numerical head and efficiency are calculated according to the energy performance equation of centrifugal pumps[19,20], which are compared with experimental data. Finally, the relatively better combination of $K_{1}$ and $K_{2}$ are obtained for the minimal discrepancy.

It can be seen form Table 2 that the simulation results with coefficients locating at middle positions are better than two extremes while $K_{1}$ changes from 0.900 to 1.100 and $K_{2}$ varies from 0.3 to 1.1 . Therefore, the scope of coefficient $K_{1}$ is set to $[0.900,1.100]$ and $K_{2}$ is adjusted to $[0.3,1.1]$. Furthermore, the results of three of them with the minimal discrepancy denoted $\mathrm{A}, \mathrm{B}$ and $\mathrm{C}$ are presented in Table 2. Finally, the values of $K_{1}$ and $K_{2}$ are given according to the range of specific speed. When it is with low specific speed, $K_{1}=1.071, K_{2}=0.9$; when it is with medium specific speed, $K_{1}=0.958, K_{2}=0.4$; when it is at high specific speed, $K_{1}=1.032, K_{2}=1.1$.

\section{Numerical verification and discussion}

\subsection{Models}

In order to verify the accuracy and reliability of the SIMPLED algorithm in centrifugal pump inner flow simulation, 3 pumps with different specific speeds were adopted. The structural parameters and experimental characteristics are shown in Table 1 for the remaining three pumps.

\subsection{Numerical methods}

Based on OpenFOAM, the inner flow of the three pumps was simulated by MRFSimpleFoam solver and MRFSimpledFoam solver with the coefficients respectively. Simultaneously, the energy performance and iteration time, then internal flow field were investigated in detail.

\subsection{Results and Analysis}

\subsubsection{Energy performance comparison}

Table 3 shows the 3 hydraulic models of centrifugal pump with different specific speeds used for the verification. Based on OpenFOAM, SIMPLE algorithm and SIMPLED algorithm are compared in terms of energy performance, iteration steps and iteration time. The results show that the iteration time by SIMPLED is almost the same as SIMPLE under the same convergence criterion, and the iteration steps by SIMPLED are slightly less than that by SIMPLE, except for high specific speed pump, which may be caused by the increased computation to a certain degree. Moreover, the calculation discrepancy of SIMPLED is much lower than that of SIMPLE. The relative discrepancy of head from SIMPLED is decreased by about $1 \% \sim 5 \%$ than that from SIMPLE, and the absolute discrepancy of efficiency also achieved similar results. All of the above indicate that SIMPLED algorithm can be applied in inner flow 
Table 3: The results of 3 pumps with SIMPLE and SIMPLED algorithms under $1.0 Q_{d}$ (S1:SIMPLED algorithm, S2: SIMPLE algorithm)

\begin{tabular}{cccccccccc}
\hline \multirow{2}{*}{ No. } & \multirow{2}{*}{ algorithms } & $\begin{array}{c}H \\
/ \mathrm{m}\end{array}$ & $\begin{array}{c}M \\
/ \mathrm{N} \cdot \mathrm{m}\end{array}$ & $\begin{array}{c}\eta_{h} \\
/ \%\end{array}$ & $\begin{array}{c}\eta \\
/ \%\end{array}$ & $\begin{array}{c}\Delta H \\
/ \%\end{array}$ & $\begin{array}{c}\Delta \eta \\
/ \%\end{array}$ & \multirow{2}{*}{ Iteration steps } & \multirow{2}{*}{ Iteration time/h } \\
\hline \multirow{2}{*}{4} & $\mathrm{~S} 1$ & 47.36 & 22.57 & 84.99 & 72.77 & 2.04 & -3.04 & 1313 & 6.36 \\
& $\mathrm{~S} 2$ & 51.37 & 26.53 & 79.37 & 68.34 & 10.68 & -7.47 & 1813 & 8.46 \\
\hline \multirow{2}{*}{5} & $\mathrm{~S} 1$ & 29.07 & 167.23 & 87.30 & 82.75 & -0.11 & 0.25 & 472 & 2.49 \\
& $\mathrm{~S} 2$ & 27.95 & 157.40 & 89.19 & 84.43 & -3.94 & 1.93 & 500 & 2.44 \\
\hline \multirow{2}{*}{6} & $\mathrm{~S} 1$ & 14.16 & 81.79 & 86.39 & 81.90 & -1.14 & -6.10 & 1002 & 5.34 \\
& $\mathrm{~S} 2$ & 15.21 & 87.69 & 86.59 & 82.23 & 6.23 & -5.77 & 993 & 5.22 \\
\hline
\end{tabular}

Table 4: The comparison of results for No. 5 pump under different flow rates by SIMPLED and SIMPLE algorithms

\begin{tabular}{|c|c|c|c|c|c|c|}
\hline \multirow{2}{*}{ experiments } & \multicolumn{6}{|c|}{ Flow rate } \\
\hline & \multicolumn{2}{|c|}{$0.8 Q_{d}$} & \multicolumn{2}{|c|}{$1.0 Q_{d}$} & \multicolumn{2}{|c|}{$1.2 Q_{d}$} \\
\hline$Q / \mathrm{m}^{3} \cdot \mathrm{h}^{-1}$ & \multicolumn{2}{|c|}{224} & \multicolumn{2}{|c|}{280} & \multicolumn{2}{|c|}{336} \\
\hline$H / \mathrm{m}$ & \multicolumn{2}{|c|}{32.00} & \multicolumn{2}{|c|}{29.10} & \multicolumn{2}{|c|}{25.40} \\
\hline$\eta / \%$ & \multicolumn{2}{|c|}{80.00} & \multicolumn{2}{|c|}{82.50} & \multicolumn{2}{|c|}{80.00} \\
\hline$n_{s}$ & \multicolumn{2}{|c|}{97.24} & \multicolumn{2}{|c|}{117.81} & \multicolumn{2}{|c|}{143.76} \\
\hline \multirow{2}{*}{ calculations } & \multicolumn{6}{|c|}{ Solving algrithm } \\
\hline & SIMPLED & SIMPLE & SIMPLED & SIMPLE & SIMPLED & SIMPLE \\
\hline$H / \mathrm{m}$ & 30.83 & 29.62 & 29.07 & 27.95 & 25.44 & 24.09 \\
\hline$M / \mathrm{N} \cdot \mathrm{m}$ & 150.49 & 163.80 & 167.23 & 157.40 & 178.59 & 169.01 \\
\hline$\eta_{h} / \%$ & 82.30 & 72.65 & 87.30 & 89.19 & 85.87 & 85.89 \\
\hline$\eta / \%$ & 79.26 & 70.11 & 82.75 & 84.43 & 81.48 & 84.45 \\
\hline$\Delta H / \%$ & -3.67 & -7.44 & -0.11 & -3.94 & 0.17 & -5.17 \\
\hline$\Delta \eta / \%$ & -0.74 & -9.89 & 0.25 & 1.93 & 1.48 & 1.45 \\
\hline Iteration steps & 668 & 1058 & 472 & 500 & 696 & 713 \\
\hline Iteration time $/ \mathrm{h}$ & 3.56 & 5.25 & 2.49 & 2.44 & 3.39 & 3.28 \\
\hline
\end{tabular}

simulation of centrifugal pump successfully and it can get better calculation accuracy than SIMPLE. Therefore, SIMPLED algorithm is a new efficient and exact modification of SIMPLE algorithm.

In order to verify further the reliability of SIMPLED algorithm, pump 5 was studied under three flow conditions. The flow rates are $0.8 Q_{d}, 1.0 Q_{d}, 1.2 Q_{d}$ respectively. In addition, the coefficients $K_{1}$ and $K_{2}$ under the three flow rates was kept the same, because the specific speed of pump 5 under those flow rates are in the range of medium specific speed. Table 4 shows experimental characteristics of pump 5 under three flow rates and the results compared with SIMPLED and SIMPLE algorithms.

It can be seen from Table 4 that although the iteration times by SIMPLED and SIMPLE are just the same under $1.0 Q_{d}$ and $1.2 Q_{d}$, the CPU time under $0.8 Q_{d}$ by SIMPLED is obviously shortened than that by SIMPLE. From the comparison of simulation data and experimental values, SIMPLED holds a better result than SIMPLE. The relative discrepancy with regards to the head under the design operating condition by SIMPLE is within -5\%, which is outside of $-5 \%$ under the remaining operating conditions. However, SIMPLED can control the relative discrepancy on $4 \%$ even little, especially under $1.0 Q_{d}$ and $1.2 Q_{d}$, the relative discrepancy of head is no more than $0.2 \%$. In addition, SIMPLED is also superior to SIMPLE in terms of efficiency. Every operating condition of efficiency gets evidently improved by SIMPLED except for $1.2 Q_{d}$. Especially under $0.8 Q_{d}$, the absolute discrepancy of efficiency by SIMPLED is within $-1 \%$, about 9 percentage points higher than that by SIMPLE.

\subsubsection{Inner flow analysis}

In order to study the adaptability of SIMPLED algorithm in depth, the impeller flow fields of pump 5 by SIMPLE algorithm and SIMPLED algorithm are given in more detail. Corresponding to the test region, the impeller passage near the volute tongue is selected by considering the interaction effect of rotor and stator. Figure 2(a) shows passage 1 is chosen for analysis. Then, three surfaces from impeller inlet to outlet are displayed on impeller meridian plane in Figure 2(b)). To avoid points falling on the solid area, a shot distance from the pressure side, suction side and impeller outlet were left, which was indicated in Figure 2(b). 5 pieces of plane curve are set in every surface, and 8 points are distributed equidistantly on each curve. All the analyses of post processing in this paper are based on those monitoring points, and points distributions are show in Figure 2(c).

(1) Static pressure distribution 


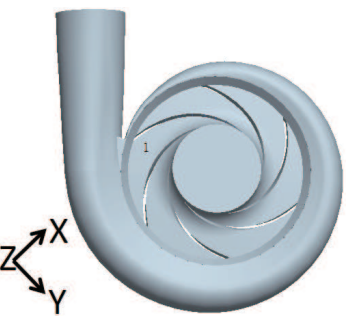

(a) Diagram of analysis passage

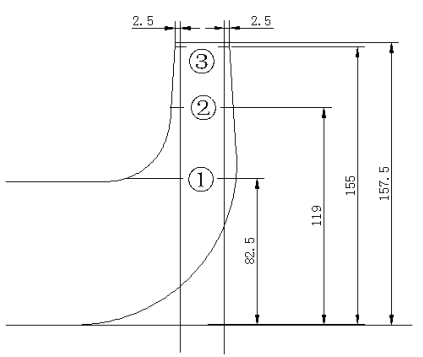

(b) Positions of analysis surfaces

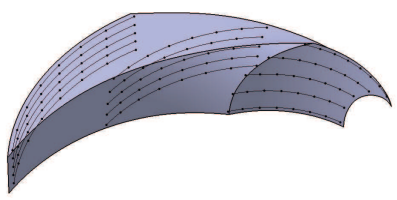

(c) Distribution of points

Fig. 2: Diagram of analysis area

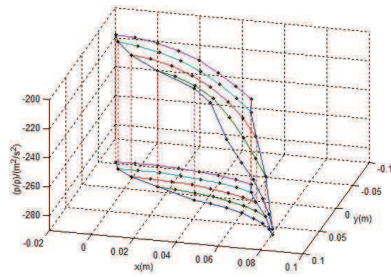

$0.8 Q_{d}$

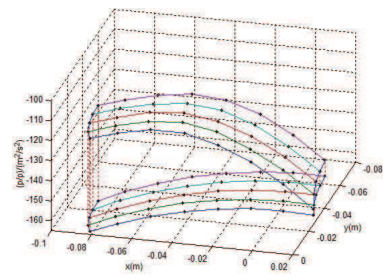

$0.8 Q_{d}$

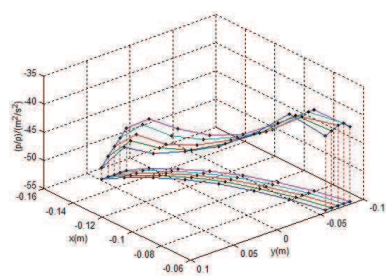

$0.8 Q_{d}$

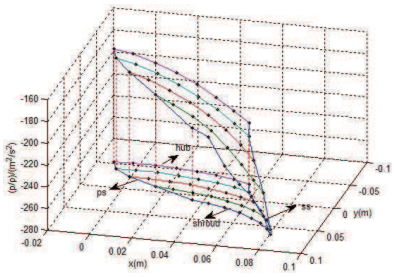

$1.0 Q_{d}$

a) surface 1

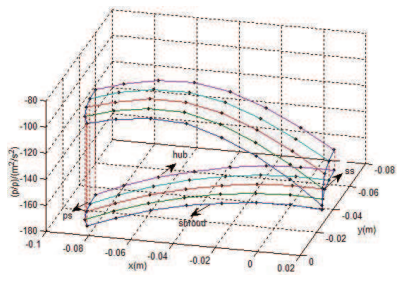

$1.0 Q_{d}$

b) surface 2

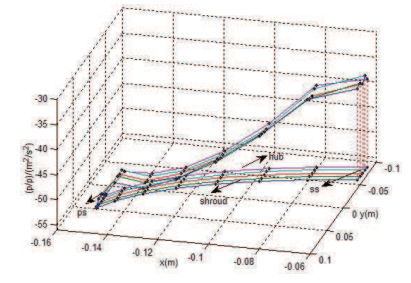

$1.0 Q_{d}$

c) surface 3

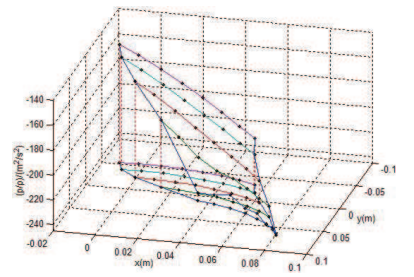

$1.2 Q_{d}$

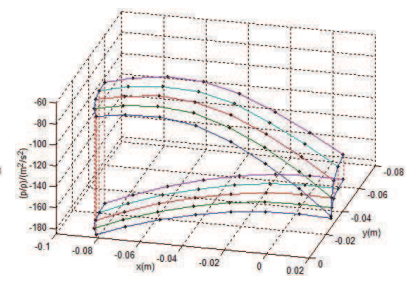

$1.2 Q_{d}$

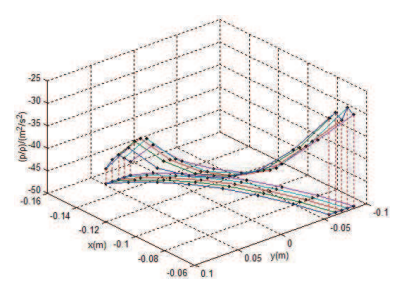

$1.2 Q_{d}$

Fig. 3: Distribution of static pressure by SIMPLE algorithm

It can be seen from Figure 3 that the static pressure distributions on surface 1 under different operating conditions are similar to each other. The static pressure of both surfaces experiences a gradually increase from the suction side to the pressure side under different flow conditions. However, there is a slight drop near the pressure side on the impeller shroud, which can be explained by the increase in the inlet velocity. Simultaneously, the static pressure under all operating conditions rises steadily from shroud to hub. Besides, the minimum value is at the suction side near the inlet. This can be interpreted that the flow is passing the leading edge of the impeller blade, it turns abruptly and the flow rate increases. Therefore, the low pressure area is formed near the impeller inlet, and then, with the rotation of impeller, the maximum value appears at the pressure side near the impeller hub. In addition, the static pressure of the flow on the surface goes up as the flow rate increases.

From Figure 3, it can be seen that the features of the static pressure distribution on surface 2 are quite similar 


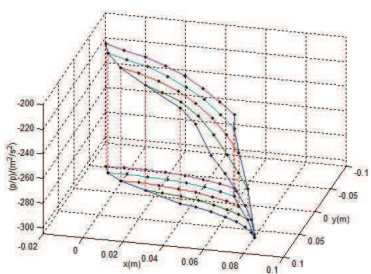

$0.8 Q_{d}$

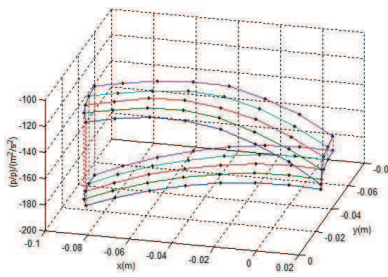

$0.8 Q_{d}$

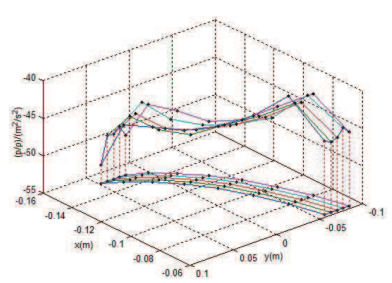

$0.8 Q_{d}$

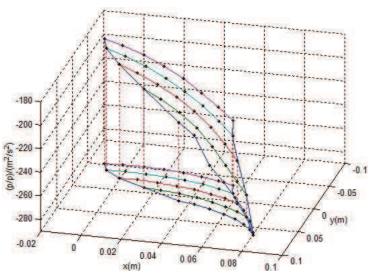

$1.0 Q_{d}$

a) surface 1

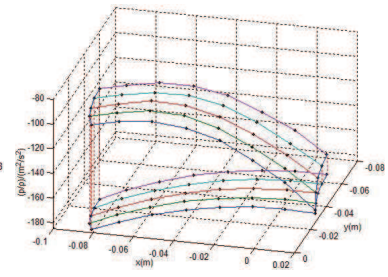

$1.0 Q_{d}$

b) surface 2

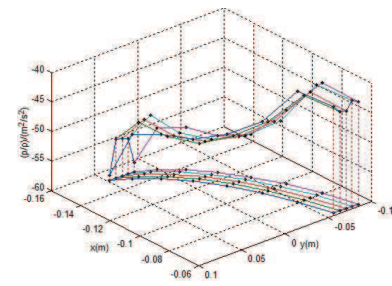

$1.0 Q_{d}$

c) surface 3

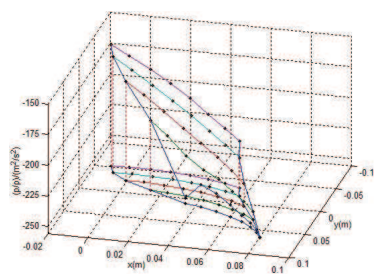

$1.2 Q_{d}$

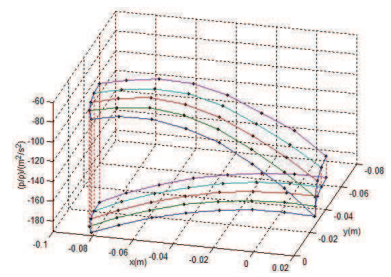

$1.2 Q_{d}$

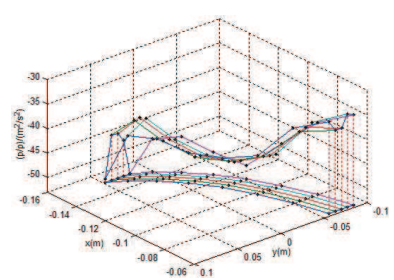

$1.2 Q_{d}$

Fig. 4: Distribution of static pressure by SIMPLED algorithm

to that on surface 1 . The difference, however, is that the static pressure distribution near the suction side on surface 2 decreases as the flow rate rises. This leads to the result that the minimum value of the static pressure under $1.2 Q_{d}$ is relatively lower than that under $0.8 Q_{d}$. This could be accounted for by the fact that with the flow rate increasing, the effect of secondary flow is reduced, and the low velocity area diminishes gradually. At the same time, the static pressure decreases as the flow velocity increases.

On surface 3 , the static pressure distribution seems a little different from other surfaces. Surface 3 lies closer to the impeller outlet and it is also close to the volute tongue. Therefore, the static pressure on surface 3 is not increasing from suction side to pressure side and from shroud to hub, but fluctuating from shroud to hub. With rising flow rate, the static pressure of corresponding points also goes up, except for the design condition. Under $0.8 Q_{d}$ and $1.0 Q_{d}$ operating conditions, the minimum value of static pressure exists near the pressure side, but the maximum value appears near the suction side. Under $1.2 \mathrm{Qd}$ condition, the minimum value of the static pressure is near the suction side and the maximum value rises near the pressure side of the hub.
In contrast, in the Figure 4, the static pressure on both surface 1 and surface 2 by SIMPLED algorithm is slightly lower than that by SIMPLE algorithm. It is suggested that the flow velocity in the impeller flow passages by SIMPLED algorithm is a litter faster than that by SIMPLE algorithm. Thus, it will give rise to dynamic pressure. Therefore, the head of pump 5 by SIMPLED algorithm is a little higher than that by SIMPLE algorithm and it is also closer to the experimental value. On surface 2 , the static pressure distribution near the suction side decreases as the flow rate rises, and it is similar to that by SIMPLE algorithm. Somewhat differently, such area is smaller than that by SIMPLE algorithm. It is because that the flow ability of the impeller by SIMPLED algorithm is better than SIMPLE algorithm and that such area becomes less prone to cavitation. On surface 3, not all the static pressures of those points are lower than that by SIMPLE algorithm. In fact, the static pressure near the pressure side is larger than that by SIMPLE algorithm under different flow conditions. It explains the fact that the flow is not easy to separate. Therefore, it will be beneficial for decreasing flow losses to a certain extent.

(2) Relative velocity distribution 


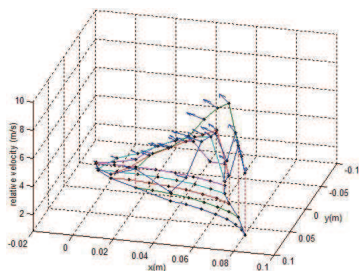

$0.8 Q_{d}$

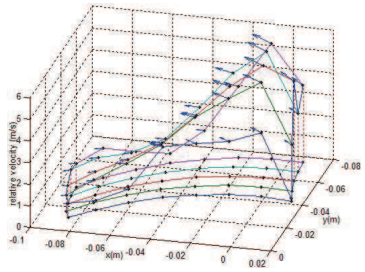

$0.8 Q_{d}$

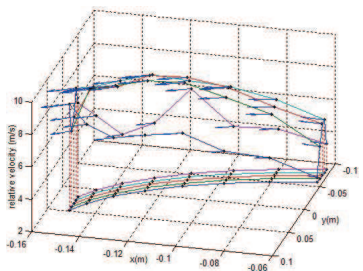

$0.8 Q_{d}$

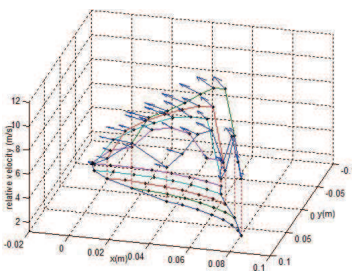

$1.0 Q_{d}$

a) surface 1

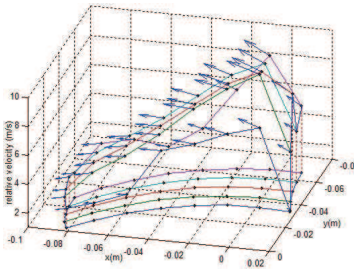

$1.0 Q_{d}$

b) surface 2

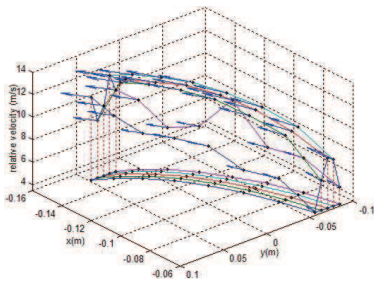

$1.0 Q_{d}$

c) surface 3

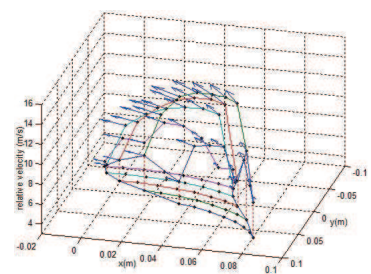

$1.2 Q_{d}$

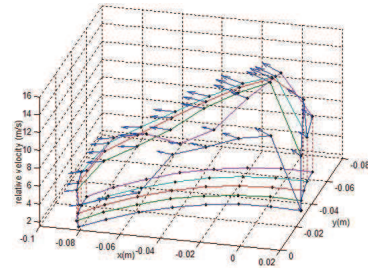

$1.2 Q_{d}$

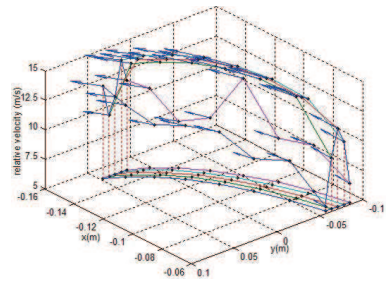

$1.2 Q_{d}$

Fig. 5: Distribution of relative velocity by SIMPLE algorithm

The relative velocity distribution of each surface under different working conditions by SIMPLE algorithm, are illustrated in Figure 5. It can be seen that the figures on surface 1 and surface 2 are quite similar. On surface 1 , the relative velocities at the same radius experiences a gradual increase before falling slightly from shroud to hub and the figures at the same circle also undergoes an increase before falling from the suction side to the pressure side. They are in agreement with the velocity distribution predicted by the potential flow theory. In addition, with the increase of flow rate, the gradients of relative velocities go up steadily. Compared with the pressure side, the values of velocities near the suction side are relatively larger. Under low flow rate, there is a low speed zone near the pressure side and the velocities grow slowly. At the $1.0 Q_{d}$ condition, the figures seem smoother and the distributions get reasonable. However, at the $1.2 Q_{d}$ condition, the low speed area near the pressure side changes slightly, but the gradients of relative velocities become larger, and it is also the area that produces the most sever backward flow situation. The distributions of relative velocity on surface 2 are much the same as that on surface 1 . However, the minimum value arises at different places. While the minimum value on surface 1 exists near the pressure side on the hub, the minimum value on surface 2 arises near the suction side on the shroud. It is generated by the fluid getting the energy from the rotation of impeller blades on the pressure side. From the distributions of relative velocities on surface 3 , the figures seem equally distributed on the middle area, but fluctuate on the shroud and hub separately. It is mainly caused by the effect of the rotor-stator interaction. With the increase in radius, the value of the relative velocity near the pressure side rises and the velocity near the suction side goes down. Finally, there is an evident feature of jet-wake structure at the impeller exit. With the increase of flow rate, the velocities of corresponding points also increase. Besides, the gap of relative velocity between the suction side and the pressure side reduces and the feature with jet-wake model becomes less significant. Meanwhile, the velocity reaches its lowest value near the suction side on the shroud, while peaking at the patch near the pressure side on the hub.

Figure 6 shows the distributions of relative velocity on different surfaces under different flow rate by SIMPLED algorithm. Compared with SIMPLE algorithm, the distributions of relative velocity simulated by SIMPLED algorithm are broadly similar to that by SIMPLE 


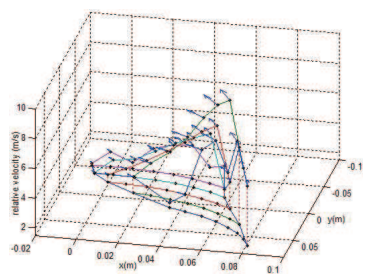

$0.8 Q_{d}$

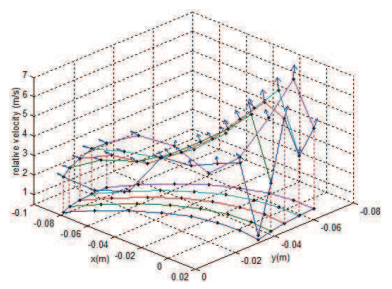

$0.8 Q_{d}$

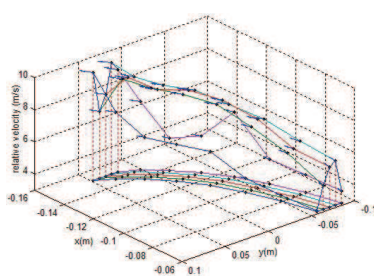

$0.8 Q_{d}$

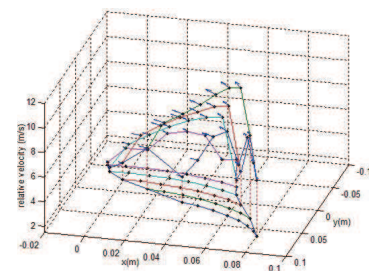

$1.0 Q_{d}$

a) surface 1

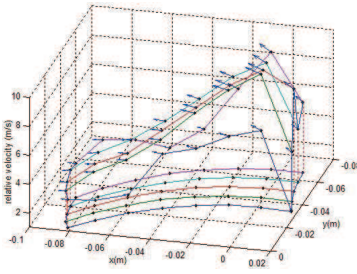

$1.0 Q_{d}$

b) surface 2

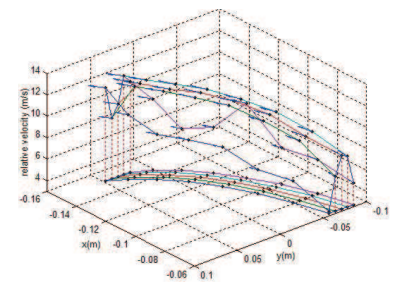

$1.0 Q_{d}$

c) surface 3

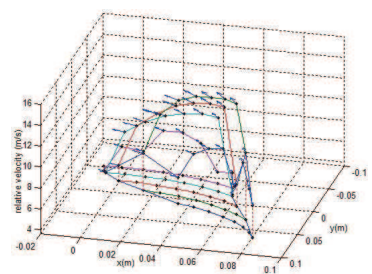

$1.2 Q_{d}$

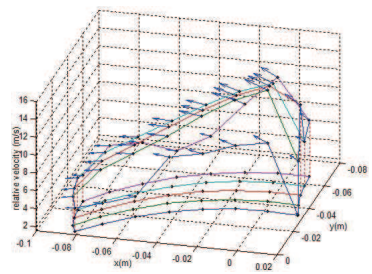

$1.2 Q_{d}$

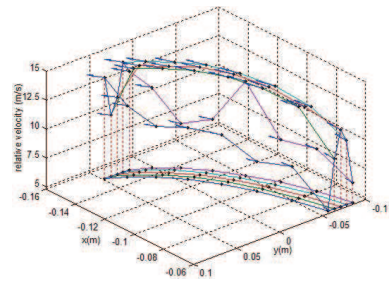

$1.2 Q_{d}$

Fig. 6: Distribution of relative velocity by SIMPLED algorithm

algorithm. The difference is that the values of relative velocity on those surfaces simulated by SIMPLED algorithm are a little larger than that by SIMPLE algorithm. It is further revealed that the head of pump 5 by SIMPLED algorithm is a little higher than that by SIMPLE algorithm.

\section{Conclusions}

In order to increase the inner flow simulation accuracy of centrifugal pump and deal with the pressure-velocity coupling in the numerical solution of N-S equations, a new modification of SIMPLE algorithm is developed for unstructured collocated grids. In this improved algorithm(SIMPLED), the pressure-correction equation appropriate for collocated grids is re-derived. Meanwhile, the cell face velocities by area weighted average method are evaluated and the pressure gradient by inverse weighted average method is calculated. The new solver is compiled using SIMPLED algorithm based on the open source code, OpenFOAM. Then, SIMPLE and SIMPLED are compared in the prediction of energy performance for 3 pumps with different specific speed under the design condition to verify the efficiency and accuracy of
SIMPLED. Lastly, the inner flow of a medium specific speed centrifugal pump is simulated with these two algorithms under the off-design conditions. Comparing the simulation data both from the energy performance and internal flow field in impeller passage, some important conclusions have been reached as follows: Firstly, under the same convergence criterion, with regards time consumption, there is no difference between SIMPLED and SIMPLE. However, SIMPLED algorithm holds a higher accuracy than SIMPLE algorithm in terms of energy performance under the design condition. Therefore, SIMPLED algorithm is a more efficient SIMPLE-like algorithm in simulation of pumps. Secondly, the inner flow under off-design conditions in a centrifugal pump with medium specific speed is studied by the two algorithms. The results show that SIMPLED is also closer to the experimental data from the energy performance than SIMPLE under the off-design conditions. Meanwhile, the pressure and relative velocity distributions in the centrifugal impeller passage for SIMPLED algorithm agree with the well known inner flow law. It is better illustrated that SIMPLED is more accurate algorithm than SIMPLE from the inner flow distribution perspective. 


\section{Perspective}

In order to increase the inner flow simulation accuracy of centrifugal pump and deal with the pressure-velocity coupling in the numerical solution of N-S equations, a new modification of SIMPLE algorithm is developed for unstructured collocated grids. In this improved algorithm(SIMPLED), the pressure-correction equation appropriate for collocated grids is re-derived. Meanwhile, the cell face velocities by area weighted average method are evaluated and the pressure gradient by inverse weighted average method is calculated. The new solver is compiled using SIMPLED algorithm based on the open source code, OpenFOAM. Then, SIMPLE and SIMPLED are compared in the prediction of energy performance for 3 pumps with different specific speed under the design condition to verify the efficiency and accuracy of SIMPLED. Lastly, the inner flow of a medium specific speed centrifugal pump is simulated with these two algorithms under the off-design conditions. Comparing the simulation data both from the energy performance and internal flow field in impeller passage, some important conclusions have been reached as follows:

Firstly, under the same convergence criterion, with regards time consumption, there is no difference between SIMPLED and SIMPLE. However, SIMPLED algorithm holds a higher accuracy than SIMPLE algorithm in terms of energy performance under the design condition. Therefore, SIMPLED algorithm is a more efficient SIMPLE-like algorithm in simulation of pumps.

Secondly, the inner flow under off-design conditions in a centrifugal pump with medium specific speed is studied by the two algorithms. The results show that SIMPLED is also closer to the experimental data from the energy performance than SIMPLE under the off-design conditions. Meanwhile, the pressure and relative velocity distributions in the centrifugal impeller passage for SIMPLED algorithm agree with the well known inner flow law. It is better illustrated that SIMPLED is more accurate algorithm than SIMPLE from the inner flow distribution perspective.

\section{Acknowledgement}

The authors gratefully acknowledge the financial support of National Natural Science Foundation of China (No. 51079062, No. 51109095, No. 51239005 and No. 51209105), Jiangsu Provincial Doctoral Innovation Foundation (No. CXZZ12_0677), Project Funded by the Priority Academic Program Development of Jiangsu Higher Education Institutions.

The authors are grateful to the anonymous referee for a careful checking of the details and for helpful comments that improved this paper.

\section{References}

[1] M. T. El-Melegy, Radial Lens Distortion Calibration from Spheres: Theory and Method, International Journal of Computing and Digital Systems, 2, 39-46 (2013).

[2] H. Benigni, H. Jaberg. H. Yeung, et al, ASME Journal of fluids engineering, 134, 024501 (2012).

[3] A. Younes, Database Manipulation Operations on Quantum Systems, Quantum Information Review, 1, 9-17 (2013).

[4] H. K. Versteeg, W. Malalasekera, An introduction to computational fluid dynamics(2nd edition) , Pearson Education Ltd, (2007).

[5] S. V. Patanker, Numerical heat transfer and fluid flow , Hemisphere publishing Corporation, Taylor\&Francis, New York, (1980).

[6] J. P. Van Doormal, G. G. Raithby, Numerical Heat Transfer, 7, 147-163 (1984).

[7] C. Hsu, A curvilinear-coordinate method for momentum, heat and mass transfer of irregular geometry, thesis, University of Minnesota, (1981).

[8] C. Prakash, A finite element method predicting flow through ducts with arbitrary cross section, thesis, University of Minnesota, (1981).

[9] C. M. Rhie, A numerical study of the flow past an isolated airfoil with separation, thesis, University of Illinois, UrbanaChampaign, (1981).

[10] C. M. Rhie, W. L. Chow, AIAA J., 21, 1525-1532 (1983).

[11] M. Peric, R. Kessler, G. Scheuerer, Computers and Fluids, 16, 389-403 (1988).

[12] S. Majumdar, Numerical Heat Transfer, 13, 125-132 (1988).

[13] A. W. Date, International Journal of Heat and Mass Transfer, 46, 4885-4898 (2003)

[14] M. H. Xu, W. Q. Tao, Journal of xi'an jiaotong university, 39, 83-86 (2005).

[15] X. H. Xiang, C. C. Su, C. H. Wang, et al, Journal of Hohai University (Natural Sciences), 36, 471-474 (2008).

[16] Harlow F. H, Welch J. E. The Physics of Fluids, 8, 21822189 (1965).

[17] A. W. Date. Numerical Heat Transfer Part B, 29, 441-458 (1996).

[18] W. Q. Tao. Numerical heat transfer(2nd edition), Xian jiaotong university press, (2000).

[19] H. L. Liu, M. G. Tan, S. Q, Welch J E. Chinese Journal of Transactions of the CSAE, 22, 107-109 (2006).

[20] X. F. Guan, Modern pumps theory and design (1st Edition), China astronautic publishing house, (2011). 


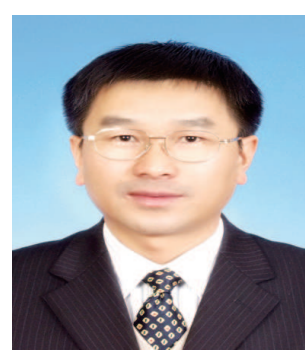

Liu Houlin received the $\mathrm{PhD}$ in Fluid Machinery Engineering from Jiangsu University. $\mathrm{He}$ is currently a Professor with Research Center of Fluid Machinery Engineering and Technology at Jiangsu University, China. He has published more than 80 papers. His research interests are in the areas of the theory, design, CAD and CFD of pumps.

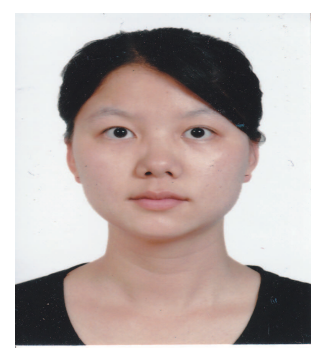

Ren Yun received the M.S degree in fluid machinery engineering from Jiangsu University. She is currently a $\mathrm{PhD}$ student in Research Center of Fluid Machinery Engineering and Technology. Her research interests are in the areas of numerical simulation of pump, SIMPLE algorithm understanding, turbulence model improvement and OpenFoam.

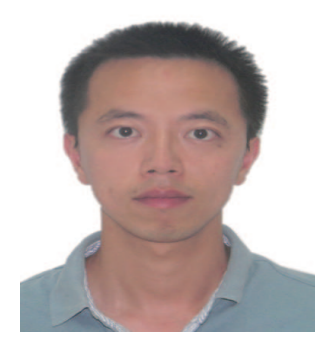

Wu Denghao received the M.S degree in fluid machinery engineering from Jiangsu University. $\mathrm{He}$ is currently a $\mathrm{PhD}$ student in Research Center of Fluid Machinery Engineering and Technology. His research interests are in the areas of design and vibration of

pumps.

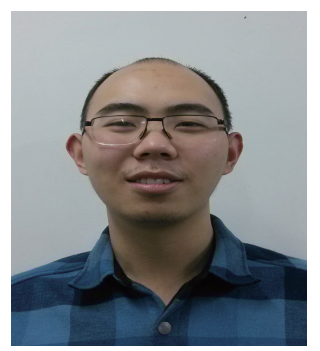

Yang Hongbin Yang Hongbin is currently a M.S candidate in Research Center of Fluid Machinery Engineering and Technology. His research interests are in the areas of experimental methods in pumps.

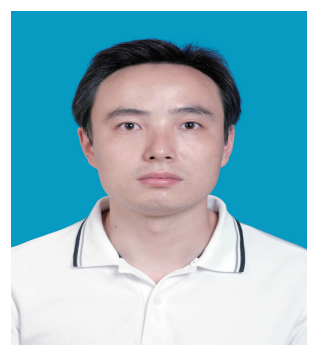

Tan Minggao Tan Minggao received the $\mathrm{PhD}$ in Fluid Machinery Engineering from Jiangsu University. $\mathrm{He}$ is currently an associate professor with Research Center of Fluid Machinery Engineering and Technology at Jiangsu University. He has published more than 20 papers. His research interests are in the areas of CFD, design and performance prediction of pumps. 